\title{
The molecular evolution of four anti-malarial immune genes in the Anopheles gambiae species complex
} Aristeidis Parmakelis*1,4, Michel A Slotman1, Jonathon C Marshall1,5, Parfait H Awono-Ambene ${ }^{2}$, Christophe Antonio-Nkondjio ${ }^{2}$, Frederic Simard ${ }^{2,3}$, Adalgisa Caccone ${ }^{1}$ and Jeffrey R Powell ${ }^{1}$

\begin{abstract}
Address: ${ }^{1}$ Department of Ecology and Evolutionary Biology, Yale University, 21 Sachem Street, 06511, New Haven, CT, USA, ${ }^{2}$ Organisation de Coordination pour la Lutte Contre les Endémies en Afrique Centrale (OCEAC), P.O. Box 288, Yaoundé, Cameroon, ${ }^{3}$ Institute de Recherche pour le Développement (IRD), UR016, BP 1857, Yaoundé, Cameroon, ${ }^{4}$ Department of Biology, University of Crete, P.O. Box 2208, GR-71409, Heraklion, Crete, Greece and 5epartment of Biology, Southern Utah University, Science Center 105, 84720, Cedar City, UT, USA

Email: Aristeidis Parmakelis* - parmakel@nhmc.uoc.gr; Michel A Slotman - michel.slotman@yale.edu;

Jonathon C Marshall - marshall@suu.edu; Parfait H Awono-Ambene - hpaawono@yahoo.fr; Christophe Antonio-

Nkondjio - antonio_nk@yahoo.fr; Frederic Simard - simard@ird.fr; Adalgisa Caccone - adalgisa.caccone@yale.edu;

Jeffrey R Powell - jeffrey.powell@yale.edu

* Corresponding author
\end{abstract}

Published: 6 March 2008

BMC Evolutionary Biology 2008, 8:79 doi:10.1186/147/-2148-8-79

This article is available from: http://www.biomedcentral.com/I47/ -2/48/8/79

(C) 2008 Parmakelis et al; licensee BioMed Central Ltd.

This is an Open Access article distributed under the terms of the Creative Commons Attribution License (http://creativecommons.org/licenses/by/2.0), which permits unrestricted use, distribution, and reproduction in any medium, provided the original work is properly cited.
Received: 14 November 2007

Accepted: 6 March 2008

\begin{abstract}
Background: If the insect innate immune system is to be used as a potential blocking step in transmission of malaria, then it will require targeting one or a few genes with highest relevance and ease of manipulation. The problem is to identify and manipulate those of most importance to malaria infection without the risk of decreasing the mosquito's ability to stave off infections by microbes in general. Molecular evolution methodologies and concepts can help identify such genes. Within the setting of a comparative molecular population genetic and phylogenetic framework, involving six species of the Anopheles gambiae complex, we investigated whether a set of four preselected immunity genes (gambicin, NOS, Rel2 and FBN9) might have evolved under selection pressure imposed by the malaria parasite.
\end{abstract}

Results: We document varying levels of polymorphism within and divergence between the species, in all four genes. Introgression and the sharing of ancestral polymorphisms, two processes that have been documented in the past, were verified in this study in all four studied genes. These processes appear to affect each gene in different ways and to different degrees. However, there is no evidence of positive selection acting on these genes.

Conclusion: Considering the results presented here in concert with previous studies, genes that interact directly with the Plasmodium parasite, and play little or no role in defense against other microbes, are probably the most likely candidates for a specific adaptive response against $P$. falciparum. Furthermore, since it is hard to establish direct evidence linking the adaptation of any candidate gene to $P$. falciparum infection, a comparative framework allowing at least an indirect link should be provided. Such a framework could be achieved, if a similar approach like the one involved here, was applied to all other anopheline complexes that transmit $P$. falciparum malaria. 


\section{Background}

Vector-borne diseases such as malaria and dengue constitute a major obstacle to socio-economic development in much of the tropics and remain high on the list of priorities for the improvement of public health. Unlike other infectious diseases, vector-borne diseases stand out because of their complex mode of transmission, requiring the transition from man to man or animal to man through an arthropod vector. This method of transmission implies the simple principle that removal of the vector will lead to the elimination of the disease [1]. This principle has been verified historically, since whenever control of an insect-borne disease has been achieved, this has most often been done through the control of the vector rather than the direct control of the disease through drugs or vaccines. The only exception has been yellow fever, for which a functional vaccine was developed early on [2]. Having this historical fact in mind, research has focused on controlling the vector, and this resulted in an increased output of medical entomological research over the last ten years or so. The best example of this is the acquisition of the complete genome sequence of Anopheles gambiae [3], the major malaria vector in sub-Saharan Africa and of Aedes aegypti [4], the major vector of yellow fever and dengue.

The wealth of results obtained has now led medical entomologists towards the development of novel ideas that take full account of this new knowledge. One such idea in which much research is invested is the "construction" of "new mosquitoes" that would be unable to transmit malaria or other diseases $[5,6]$. This "new mosquito" would be based on transgenic strains carrying genes that make them refractory to their parasites. After almost two decades in effort, the technology for creating transgenic mosquitoes has been developed and encouraging results have sprouted from this research [7-9]. However, there is still the need to identify those refractory genes best suited for "constructing" transgenic mosquitoes.

In the case of malaria, the insect's immunity genes have been considered as very good candidates, since following the sequencing of the An. gambiae genome [3] a large set of genes that mediate mosquito susceptibility to Plasmodium infections has been identified [10-16]. However, if the insect innate immune system is to be used as a potential blocking step in transmission of malaria, then it will require targeting one or a few genes of highest relevance and ease of manipulation. The problem, then, is to identify those of highest importance to malaria infection. By and large, the immune responses are adaptive for the mosquito. Therefore, modification of such genes runs the risk of decreasing the mosquito's ability to stave off infections by microbes in general, thereby decreasing mosquito fitness and lessening the chance to become established in the natural population. Ideally, one would want to identify a gene, or part of a gene, that specifically targets the particular pathogen of interest. Furthermore, it is crucial that the gene(s) used in any attempt to block transmission in natural populations focus on genes specific to the relevant mosquito and Plasmodium species, since it has been suggested that the defense exhibited by the vector species, varies depending on the invading Plasmodium species [11].

In this study we focus on the species of the An. gambiae complex and we assess the genetic polymorphism of four genes (gambicin, NOS, Rel2 and FBN9) that have been identified as part of the innate immune system of An.gambiae. The An. gambiae complex is composed of seven closely related species, i.e. An. gambiae, Anopheles arabiensis, Anopheles melas, Anopheles merus, Anopheles bwambae and Anopheles quadriannulatus A and B. The first two species are the major vectors of human malaria in sub-Saharan Africa, with An. melas and An. merus being intermediate in importance. The last three species are highly zoophilic and are never or rarely exposed to the human Plasmodium falciparum.

The studied loci have been implicated in the resistance towards Plasmodium infections largely through microarray and RNAi experiments [11,17-19]. Molecular population genetics and phylogenetics is an independent approach that could verify that evolutionary patterns re-enforce implications from laboratory studies. Within a comparative framework, we evaluate the possibility that these four genes evolve, in the lineages of the major malaria vectors, under selection imposed by the direct or indirect interaction with the Plasmodium. The approach relies on the prediction that if Plasmodium infection affects the mosquito's fitness, we may expect the accumulation of adaptive amino acid substitutions in those anti-malarial genes that are crucial in specifically limiting Plasmodium infection in vector species, whereas such changes are less likely to be found in closely related species that have historically limited interaction with the parasite. An assumption of this approach is that the Anopheles species have exhibited an adaptive response to P.falciparum infection which is suggested by several lines of evidence [20].

\section{Results}

\section{Polymorphism, divergence and McDonald-Kreitman tests} Gambicin

A fragment of 589 to 682 bp (Table 1) was amplified from all specimens. This fragment includes all the coding sequence of the gene, consisting of $246 \mathrm{bp}$. A total of 59 sequences (Table 2) including the whole coding region of this gene were obtained from six species of the An. gambiae complex. Out of the 59 sequences 37 represented different alleles. The nucleotide diversity (Pi) varied from 
Table I: Sequences of primers used in the study for the amplification of the four anti-malarial immunity genes. If PCR, was not succesfull or produced very low signal a nested PCR was applied.

\begin{tabular}{|c|c|c|}
\hline & $P C R_{I}$ & $P C R_{\text {nested }}$ \\
\hline \multirow[t]{4}{*}{ Gambicin } & Gamb_exon_313_I505F & Gamb_exon_46_692F \\
\hline & TGAATCCCCTCGGCTCGCTG & CTGAACGCCGTCACAAGTGC \\
\hline & Gamb_exon_313_I505R & Gamb_exon_46_692R \\
\hline & TGCAGTGAGTTATGTCACAAGC & TGGCACTGATTAAACCGCTTG \\
\hline \multirow[t]{4}{*}{ NOS } & NOS_exon_30528F & NOS_exon_30705F \\
\hline & GTGGAYGGAATYATYGAGCG & GGTGTCTACAAATCKGGGA \\
\hline & NOS_exon_31858R & NOS_exon_31692R \\
\hline & MCGCSYTACTTACCCGCAGCG & CGAKTCCGCCTCYTTGAGGGC \\
\hline \multirow[t]{4}{*}{ Rel2 } & Rel2_exon_4I5F & Rel2_exon_504F \\
\hline & ACACCGTCCTGTCGATGGAC & GGTCGCACCTATGCCAGTGC \\
\hline & Rel2FragIRev & Rel2_exon_I275R \\
\hline & GATGCCCATACCCTGGAAGG & ACACCCTCCGATGGTTCAGC \\
\hline \multirow[t]{4}{*}{ FBN9 } & FBN9_2I7F & FBN9_264F \\
\hline & TCCGACCTCCACCGGGTACG & ACTACCTACAGTACAAGCTGCTC \\
\hline & FBN9_II49R & FBN9_I075R \\
\hline & AGCCATGCCCTGGTGCGAGC & GGCAGTTGTTGTACCACCAG \\
\hline
\end{tabular}

0.000 to 0.024 and 0.001 to 0.007 in the synonymous and non-synonymous sites, respectively (Table 2). There were very few mutations (4 mutations in 3 pairwise comparisons) shared between species, whereas there were four alleles out of the 37, that were shared between them. For the coding region, Dxy (average number of nucleotide substitutions per site between species) ranged from 0.006 to 0.014 . Very few fixed differences were present between species, and in most comparisons no fixed non-synonymous differences were found (Table 3). Not surprisingly therefore, the McDonald-Kreitman tests did not indicate positive selection.

\section{NOS}

A total of 46 sequences were produced from the studied species (Table 2). The sequences produced varied from 993 to $1007 \mathrm{bp}$, of which $771 \mathrm{bp}$ were coding. Out of the 46 sequences 38 were different alleles. Although there were some shared polymorphisms (10 in six pairwise comparisons) between the species, there were no alleles shared between them. Dxy ranged from 0.009 to 0.017 for the coding region. Nucleotide diversity $(\mathrm{Pi})$ ranged between 0.007 to 0.050 and 0.001 to 0.003 in the synonymous and non-synonymous sites, respectively. A single fixed replacement substitution was observed in all pairwise comparisons (Table 3) with An. arabiensis (except with An. gambiae). However, none of the McDonald-Kreitman tests were significant.

\section{Rel2}

We obtained 74 sequences from six species. The fragments amplified in all specimens ranged from 665 to $803 \mathrm{bp}$. Of these approximately 710 bp were coding sequence (Table 2 ). Out of the 74 sequences 49 were different alleles (Table 2). There were a few polymorphisms shared between species (nine in two pairwise comparisons) but there were no shared alleles. Dxy ranged from 0.014 to 0.037 between species. The nucleotide diversity $(\mathrm{Pi})$ in the synonymous as well as in the non-synonymous sites was quite low varying from 0.000 to 0.031 in the synonymous sites, and from 0.000 to 0.004 in the non-synonymous sites. As was the case in the previous genes, the McDonaldKreitman tests of positive selection did not show a significant excess of fixed non-synonymous differences between the species, although there were fixed replacement substitutions present in all the pairwise species comparisons (Table 4).

FBN9

We successfully determined 60 sequences from the six species all together. The fragments amplified in all specimens ranged from 764 to $807 \mathrm{bp}$, and they were all coding sequence (Table 2). Of these 60 sequences, 54 were different alleles (Table 2). There were a lot of mutations (48 mutations in 10 pairwise comparisons) shared between species, but again there were no alleles shared. Dxy ranged from 0.016 to 0.029 between species. Nucleotide diversity (Pi) in the synonymous sites was higher compared to the previous genes and varied from 0.016 to 0.079 . However, it was quite low in the non-synonymous sites since it ranged from 0.000 up to 0.003 . As presented in Table 4 there is not even a single fixed non-synonymous substitution between the species of the complex. Consequently, 
Table 2: Sequence data and polymorphism parameters of the four amplified immunity genes in the species of the An. gambiae complex. Length of sequences, exons and number of exons refer to the fragments sequenced in this study. Alleles were inferred based on the coding regions of the sequences.

\begin{tabular}{|c|c|c|c|c|c|c|c|c|}
\hline & \multirow{2}{*}{$\begin{array}{l}\text { Number of } \\
\text { individuals }\end{array}$} & \multirow{2}{*}{$\begin{array}{l}\text { Length of } \\
\text { sequences } \\
\text { obtained (bp) }\end{array}$} & \multirow{2}{*}{$\begin{array}{l}\text { Number of } \\
\text { exons/length } \\
\text { of each exon } \\
\text { sequence (bp) } \\
\text { analysed }\end{array}$} & \multirow{2}{*}{$\begin{array}{l}\text { Number of } \\
\text { sequences } \\
\text { obtained per } \\
\text { species } \\
\text { (number of } \\
\text { alleles per } \\
\text { species) }\end{array}$} & \multicolumn{2}{|c|}{ Polymorphic sites } & \multicolumn{2}{|c|}{ Nucleotide diversity (Pi) } \\
\hline & & & & & Syn. & Non-Syn. & Syn. & Non-Syn. \\
\hline \multicolumn{9}{|l|}{ Gambicin } \\
\hline An. arabiensis & 6 & $646-677$ & $3 / 75,90,81$ & $10(8)$ & 5 & 5 & 0.002 & 0.007 \\
\hline An. bwambae & 6 & $667-675$ & $3 / 75,90,81$ & II (7) & 4 & 5 & 0.020 & 0.006 \\
\hline An. gambiae & 5 & $666-672$ & $3 / 75,90,81$ & $9(8)$ & 4 & 4 & 0.024 & 0.006 \\
\hline An. melas & 6 & $589-672$ & $3 / 75,90,81$ & $11(4)$ & 0 & 5 & 0.000 & 0.004 \\
\hline An. merus & 5 & $664-682$ & $3 / 75,90,81$ & $8(4)$ & 2 & 1 & 0.008 & 0.001 \\
\hline $\begin{array}{l}\text { An. } \\
\text { quadriannulatus }\end{array}$ & 5 & $663-682$ & $3 / 75,90,81$ & $10(6)$ & 5 & 4 & 0.019 & 0.004 \\
\hline Total & 33 & & & $59(37)$ & & & & \\
\hline \multicolumn{9}{|l|}{ NOS } \\
\hline An. arabiensis & 5 & $997-1006$ & $3 / 201,432,138$ & $5(5)$ & 19 & 2 & 0.050 & 0.001 \\
\hline An. bwambae & 7 & $997-1008$ & $3 / 201,432,138$ & $11(8)$ & 10 & 7 & 0.021 & 0.003 \\
\hline An. gambiae & 6 & $1006-1007$ & $3 / 201,432,138$ & $7(7)$ & 16 & 6 & 0.029 & 0.003 \\
\hline An. melas & 5 & $1004-1007$ & $3 / 201,432,138$ & $8(5)$ & 3 & 4 & 0.007 & 0.002 \\
\hline An. merus & 6 & $993-1001$ & $3 / 201,432,138$ & $10(8)$ & 15 & 4 & 0.028 & 0.001 \\
\hline $\begin{array}{l}\text { An. } \\
\text { quadriannulatus }\end{array}$ & 5 & $1002-1006$ & $3 / 201,432,138$ & $5(5)$ & 7 & 2 & 0.019 & 0.001 \\
\hline Total & 34 & & & $46(38)$ & & & & \\
\hline \multicolumn{9}{|l|}{ Rel2 } \\
\hline An. arabiensis & 10 & $780-785$ & 2/372, 339 & $20(18)$ & 15 & 10 & 0.031 & 0.004 \\
\hline An. bwambae & 6 & $712-778$ & 2/372, 339 & $6(1)$ & 0 & 1 & 0.000 & 0.000 \\
\hline An. gambiae & 14 & $732-794$ & 2/372, 339 & $16(11)$ & 19 & 6 & 0.020 & 0.004 \\
\hline An. melas & 8 & $761-800$ & $2 / 372,339$ & $10(5)$ & 3 & 2 & 0.008 & 0.001 \\
\hline An. merus & 7 & $665-796$ & 2/372, 339 & $11(6)$ & 2 & 4 & 0.003 & 0.002 \\
\hline $\begin{array}{l}\text { An. } \\
\text { quadriannulatus }\end{array}$ & 8 & $762-803$ & 2/372, 339 & $11(8)$ & 9 & 4 & 0.013 & 0.002 \\
\hline Total & 53 & & & $74(49)$ & & & & \\
\hline \multicolumn{9}{|l|}{ FBN9 } \\
\hline An. arabiensis & 7 & $789-807$ & I/807 & $12(12)$ & 40 & 7 & 0.065 & 0.003 \\
\hline An. bwambae & 7 & $787-807$ & $1 / 807$ & $8(3)$ & 18 & 2 & 0.040 & 0.001 \\
\hline An. gambiae & 7 & $764-807$ & $1 / 807$ & $12(12)$ & 41 & 1 & 0.079 & 0.000 \\
\hline An. melas & 7 & 776-79| & I/79| & $7(7)$ & 9 & 0 & 0.021 & 0.000 \\
\hline An. merus & 7 & $788-807$ & $1 / 807$ & $11(10)$ & 10 & 4 & 0.016 & 0.002 \\
\hline $\begin{array}{l}\text { An. } \\
\text { quadriannulatus }\end{array}$ & 7 & $780-807$ & $\mathrm{I} / 807$ & $10(10)$ & 18 & 6 & 0.034 & 0.002 \\
\hline Total & 42 & & & $60(54)$ & & & & \\
\hline
\end{tabular}


the McDonald-Kreitman tests were negative regarding positive selection acting on the gene (Table 4).

A second series of McDonald-Kreitman tests were performed for all the genes, but this time the alleles were not grouped according to species. They were grouped according to their phylogenetic relationships, as indicated by the inferred phylogenetic trees. For example in Rel2, a set of the An. gambiae alleles were pooled together with the An. bwambae sequences to form a single group, as indicated by the phylogenetic tree of figure 1 . This group of alleles was subsequently contrasted to the alleles of the remaining species. Again no signs of positive selection could be detected.

\section{Phylogeny and maximum likelihood tests for selection}

Since no non-synonymous fixed differences were found in any species pairwise comparisons for Gambicin and FBN9 (Table 3,4) only NOS and Rel2 were subjected to the PAML analysis. The phylogenetic trees for these two genes, produced from the bayesian analysis, and on which the various models of the PAML software were evaluated for their goodness of fit, are presented in figures 1 and 2.

In the NOS tree (Figure 2) only An. merus, An. melas and An. quadriannulatus form strongly supported monophyletic groups. Some alleles of An.bwambae (BWA16 B/ BWA12 B and BWA11 A/BWA11 B) are placed very far apart from their conspecific sequences. Furthermore, each one of these two sets of An. bwambae alleles, seems to be closely related to a different Anopheles species. The alleles
BWA16 B/BWA12 B are placed within the clade hosting An. merus, An. bwambae and An. quadriannulatus, whereas the alleles BWA11 A/BWA11 B seem to be more closely related to An. gambiae and An. arabiensis. At the same time, the alleles of An. gambiae seem to be forming two separate clades that are firmly to each other and are ambiguously related to the An. arabiensis clade.

In the Rel2 tree (Figure 1), the alleles of most species form strongly supported monophyletic groups and the relationships between the species are well resolved (posterior probabilities above 0.80). As was the case in NOS, the alleles of An. gambiae form two separate clades that are placed apart. In the case of Rel2, one of the An. gambiae groups is closely related to An. bwambae, and the other is part of a broader clade that incorporates both the $A n$. merus and the An. melas clades.

In the case of the NOS gene, only the likelihood ratio test of $M 0$ versus $M 3$ (Table 5) was statistically significant and in favor of variable selection pressure among sites. All other likelihood ratio tests comparing the among sites models, were not in favor of an $\omega$ value greater than one among sites (Table 5). Similar results were obtained for Rel2, where again only the comparison between models $\mathrm{M} 0$ and M3 (Table 5) indicated variable selection pressure acting on the amplified Rel2 fragment.

Regarding the branch-site tests for the NOS and Rel2 (Table 6), regardless of whether An. gambiae or An. arabiensis were designated as the foreground branches, the like-

Table 3: MacDonald-Kreitman tests on gambicin and NOS.

\begin{tabular}{|c|c|c|c|c|c|c|c|c|c|c|}
\hline & \multicolumn{5}{|c|}{ Gambicin } & \multicolumn{5}{|c|}{ NOS } \\
\hline & \multicolumn{2}{|c|}{ Fixed } & \multicolumn{2}{|c|}{ Polymorp. } & \multirow[b]{2}{*}{$\mathrm{p}$-value } & \multicolumn{2}{|c|}{ Fixed } & \multicolumn{2}{|c|}{ Polymorp. } & \multirow[b]{2}{*}{$\mathrm{P}$-value } \\
\hline & $S$ & NS & $S$ & NS & & $S$ & NS & $S$ & NS & \\
\hline gam-ara & 0 & 0 & 9 & 8 & -- & 1 & 0 & 33 & 8 & n.s. \\
\hline gam-qua & 0 & 0 & 9 & 8 & -- & 2 & 0 & 22 & 8 & n.s. \\
\hline gam-mel & I & 0 & 4 & 9 & n.s. & 2 & 0 & 17 & 9 & n.s. \\
\hline gam-mer & 0 & 0 & 6 & 5 & -- & 2 & 0 & 29 & 10 & n.s. \\
\hline gam-bwa & 0 & 0 & 8 & 9 & -- & 1 & 0 & 24 & 13 & n.s. \\
\hline ara-qua & 0 & 0 & 9 & 8 & -- & 2 & 1 & 25 & 4 & n.s. \\
\hline ara-mel & 0 & 0 & 5 & 10 & -- & 3 & 1 & 22 & 5 & n.s. \\
\hline ara-mer & 0 & 0 & 7 & 5 & -- & 1 & 1 & 31 & 6 & n.s. \\
\hline ara-bwa & 0 & 0 & 9 & 10 & -- & 0 & I & 29 & 9 & n.s. \\
\hline qua-mel & 0 & 0 & 5 & 9 & -- & 3 & 0 & 10 & 6 & n.s. \\
\hline qua-mer & 0 & 0 & 7 & 5 & -- & 1 & 0 & 22 & 6 & n.s. \\
\hline qua-bwa & 0 & 0 & 9 & 9 & -- & 1 & 0 & 15 & 9 & n.s. \\
\hline mel-mer & I & 0 & 2 & 6 & n.s. & 3 & 0 & 17 & 7 & n.s. \\
\hline mel-bwa & I & 0 & 4 & 10 & n.s. & 2 & 0 & 13 & 10 & n.s. \\
\hline mer-bwa & 0 & 0 & 6 & 6 & -- & 0 & 0 & 23 & 11 & -- \\
\hline
\end{tabular}

Species names are abbreviated as follows: An. arabiensis: ara, An. bwambae: bwa, An. gambiae: gam, An. melas: mel, An. merus: mer, An. quadriannulatus: qua. S: synonymous mutations, NS: non-synonymous mutations, n.s.: non significant. 
Table 4: MacDonald-Kreitman tests on Rel2 and FBN9.

\begin{tabular}{|c|c|c|c|c|c|c|c|c|c|c|}
\hline & \multicolumn{5}{|c|}{ Rel2 } & \multicolumn{5}{|c|}{ FBN9 } \\
\hline & \multicolumn{2}{|c|}{ Fixed } & \multicolumn{2}{|c|}{ Polymorp. } & \multirow[b]{2}{*}{$\mathrm{P}$-value } & \multicolumn{2}{|c|}{ Fixed } & \multicolumn{2}{|c|}{ Polymorp. } & \multirow[b]{2}{*}{$\mathrm{P}$-value } \\
\hline & $\mathrm{S}$ & NS & $\mathrm{S}$ & NS & & $\mathrm{S}$ & NS & $\mathrm{S}$ & NS & \\
\hline gam-ara & I & 0 & 26 & 13 & n.s. & 0 & 0 & 63 & 7 & -- \\
\hline gam-qua & 2 & I & 24 & 9 & n.s. & 3 & 0 & 57 & 7 & n.s. \\
\hline gam-mel & I & 2 & 22 & 8 & n.s. & 5 & 0 & 48 & 1 & n.s. \\
\hline gam-mer & I & 1 & 18 & 8 & n.s. & 3 & 0 & 49 & 5 & n.s. \\
\hline gam-bwa & 2 & 2 & 18 & 6 & n.s. & 2 & 0 & 52 & 2 & n.s. \\
\hline ara-qua & 2 & I & 20 & 12 & n.s. & 2 & 0 & 55 & 13 & n.s. \\
\hline ara-mel & 7 & 8 & 17 & 11 & n.s. & 6 & 0 & 49 & 7 & n.s. \\
\hline ara-mer & 2 & 1 & 16 & 12 & n.s. & 3 & 0 & 49 & II & n.s. \\
\hline$a r a-b w a$ & 5 & 2 & 15 & 10 & n.s. & 4 & 0 & 51 & 8 & n.s. \\
\hline qua-mel & 6 & 7 & II & 6 & n.s. & 9 & 0 & 26 & 6 & n.s. \\
\hline qua-mer & 4 & 3 & 10 & 7 & n.s. & 4 & 0 & 26 & 10 & n.s. \\
\hline qua-bwa & 4 & 3 & 8 & 5 & n.s. & 7 & 0 & 34 & 8 & n.s. \\
\hline mel-mer & 3 & I & 5 & 6 & n.s. & 13 & 0 & 19 & 4 & n.s. \\
\hline mel-bwa & II & 6 & 3 & 3 & n.s. & 5 & 0 & 26 & 2 & n.s. \\
\hline mer-bwa & 9 & 2 & 2 & 4 & n.s. & 5 & 0 & 28 & 6 & n.s. \\
\hline
\end{tabular}

Species names are abbreviated as in Table 3. S: synonymous mutations, NS: non-synonymous mutations, n.s.: non significant.

lihood ratio tests were not in favor of positive selection acting on at least some codons of the foreground branches in either gene. The branch-site test 2 for NOS, was also applied to a phylogenetically designated "gambiae" clade. By this we mean that we considered as the gambiae clade (foreground branch) not only the alleles of An. gambiae, but those of An. arabiensis and An. bwambae that are ambiguously related to the An. gambiae alleles, as well (Figure 2). The result of the branch-site test 2 was negative in this case as well.

\section{Discussion}

The nucleotide diversity (Pi) of all studied loci was quite low in all the species of the An. gambiae complex both in the synonymous and the non-synonymous substitutions (Table 2). Gambicin exhibited the lowest levels of variation and FBN9 the least (Table 2). The levels of divergence of these loci between the different species were low as well. Among the four analyzed loci, Rel2 and FBN9 were the most divergent, with Dxy values range from 1.4 to $3.7 \%$ and 1.6 to $2.9 \%$, respectively.

In the gambicin locus it can be seen that in some species pairwise comparisons the fixed differences (synonymous and non-synonymous) are zero (Table 3 ). One could argue that this could be the result of introgression between the species and/or the continued segregation of inherited ancestral polymorphisms. However, we argue that these two processes have not equally affected the observed pattern. Introgression cannot be ruled out between the lineages of An. gambiae and An. arabiensis, since in the phylogenetic tree of the respective gene (see
Additional file 1), the alleles of An. arabiensis cluster together with the An. gambiae alleles. Evidence for introgression between An. gambiae and An. arabiensis has been reported previously [20-23]. At the same time, the role of the shared ancestral polymorphisms in the observed pattern is limited since in gambicin the shared polymorphisms between the species were in the majority of the pairwise comparisons zero (see results). We estimated (results not shown) the level of divergence between species using the non-coding regions of the gambicin gene. The net between species average Kimura two-parameter distance [24] as estimated by MEGA v.3.1 [25], ranged between $0.4 \%$ (An. gambiae versus $A n$. arabiensis) and $3.8 \%$ (An. melas versus An. arabiensis). This level of divergence is quite low compared to the respective level of divergence for Rel2 and NOS (see below). The complete absence of fixed non-synonymous substitutions between pairwise species comparisons in the gambicin locus indicates that purifying selection is the major force that shapes the observed evolutionary pattern of gambicin (Table 3 ). Gambicin has been found to be an important antibacterial peptide, that is however, marginally lethal to $P$. berghei [19] and has no specificity towards P. falciparum [11]. More likely, this gene as many others belonging to the antimicrobial defense system of the mosquito, evolve under the selection constrains imposed by the bacteria that the mosquitoes encounter during their lifetime. As Dong et al. (2006) also conclude there is little reason to believe that gambicin has undergone major adaptations to malaria. 


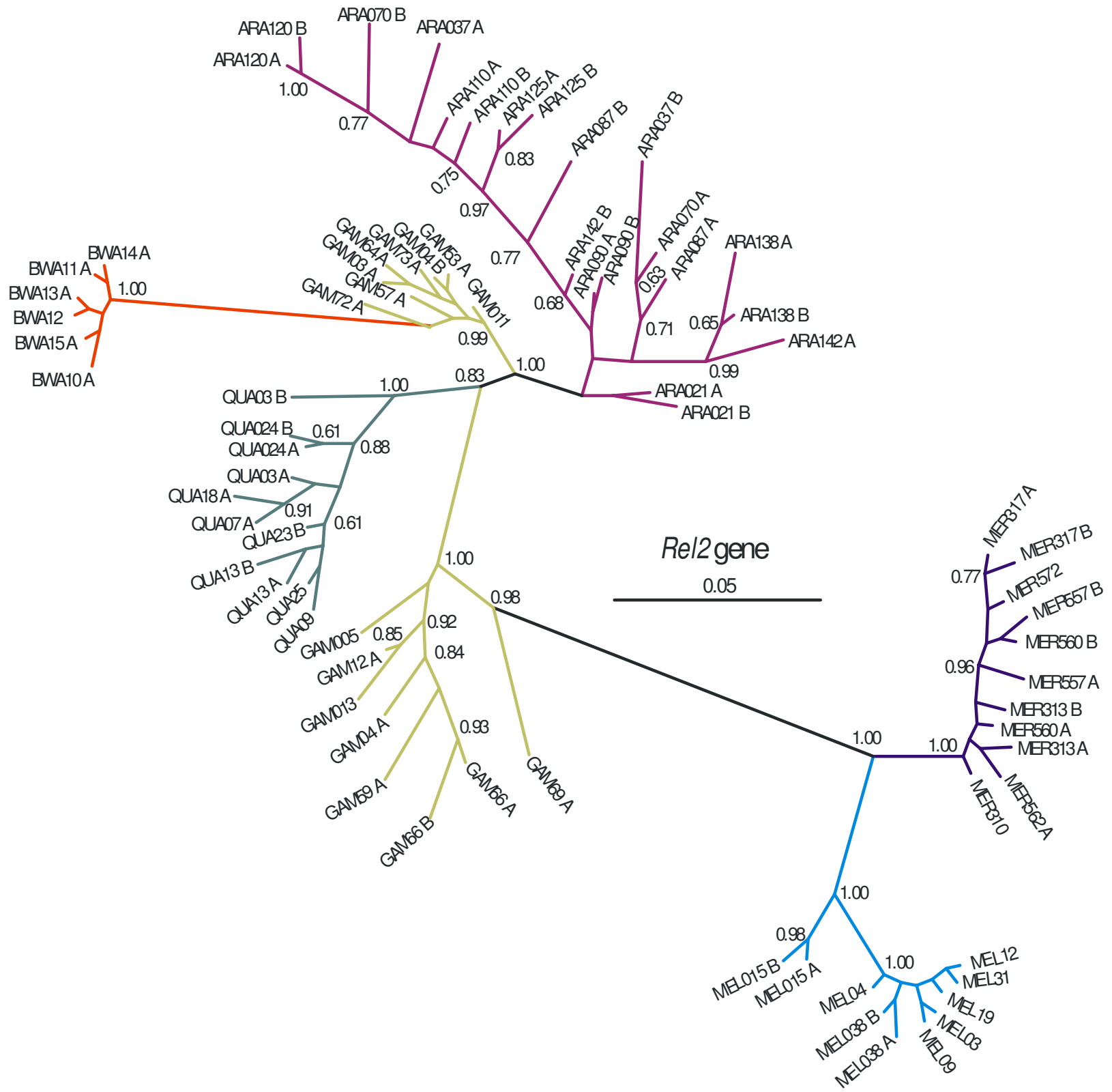

\section{Figure I}

Rel2 Bayesian Inference Tree. 50\% majority-rule consensus Bayesian (unrooted) tree of Rel2. Numbers on branches are the posterior probabilities of clades, only values above 0.5 are presented. Species names have been abbreviated as follows: ARA: An. arabiensis, BWA: An. bwambae, GAM: An. gambiae, MEL: An. melas, MER: An. merus, and QUA: An. quadriannulatus. The number following the species abbreviation refers to the individual specimen code, whereas the letters $A$ and $B$ differentiate between the two alleles of a single individual specimen. Details of the Bayesian analysis can be provided upon request.

In the NOS locus, a great number of polymorphic sites were recorded in all species pairwise comparisons (Table 3). However, the fixed non-synonymous difference was one in each species comparison of An. arabiensis with the other species (except An. gambiae). As was the case with the gambicin locus, in the phylogenetic tree of NOS, signs of introgression between An. gambiae and An. arabiensis are evident (Figure 2). Moreover, two alleles originating from An. bwambae, were placed within the An. gambiael An. arabiensis clade, another two alleles were placed very 


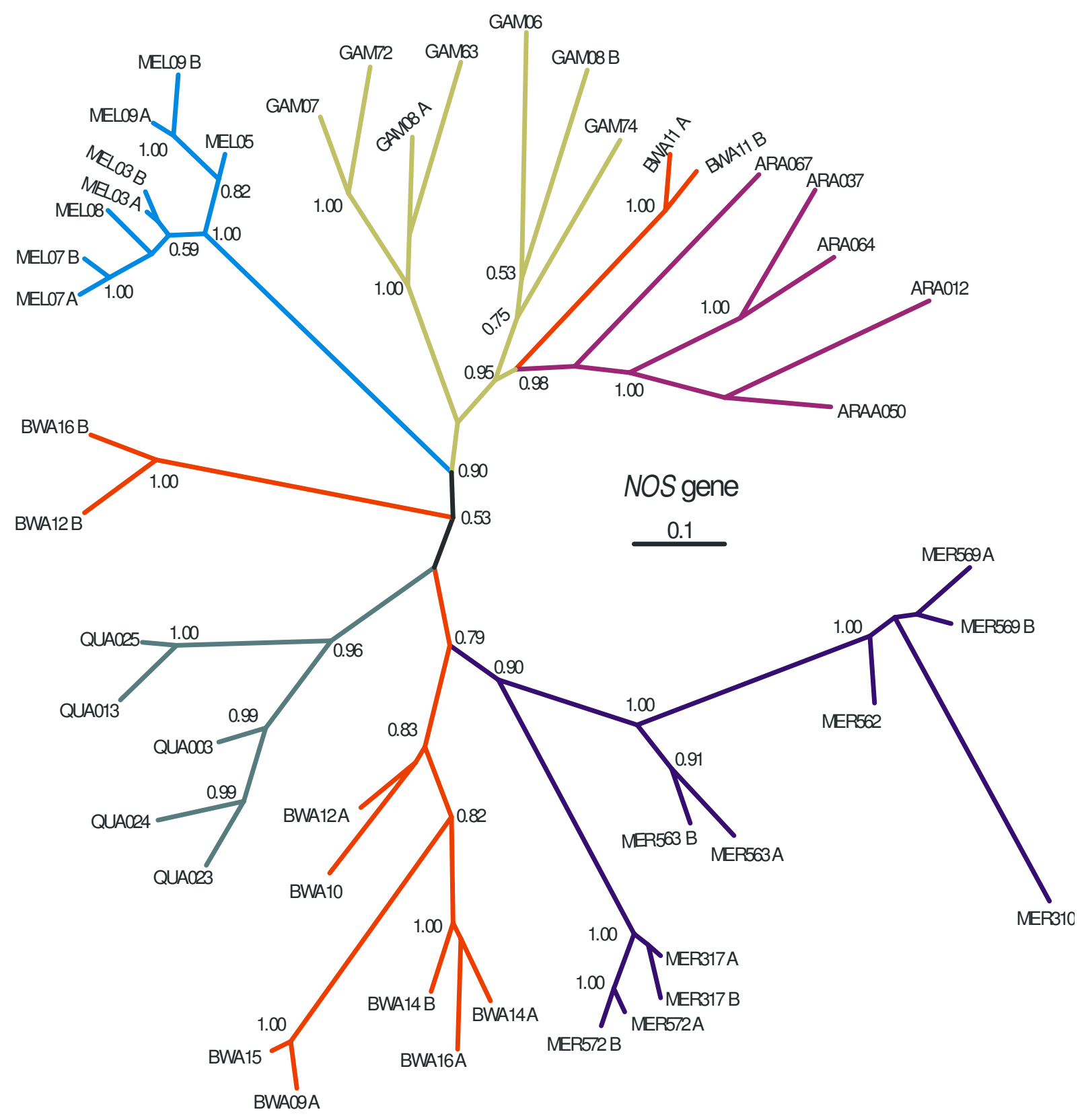

Figure 2

NOS Bayesian Inference Tree. 50\% majority-rule consensus Bayesian (unrooted) tree of NOS. Numbers on branches are the posterior probabilities of clades, only values above 0.5 are presented. Species names have been abbreviated as follows: ARA: An. arabiensis, BWA: An. bwambae, GAM: An. gambiae, MEL: An. melas, MER: An. merus, and QUA: An. quadriannulatus. The number following the species abbreviation refers to the individual specimen code, whereas the letters $A$ and $B$ differentiate between the two alleles of a single individual specimen. Details of the Bayesian analysis can be provided upon request.

close to An. melas and the majority of the alleles formed a separate and relatively well supported monophyletic clade. The case for genetic introgression between $A n$. bwambae and An. gambiae has been made previously [26], and this is reflected here as well. However, we consider that in the case of NOS, we are also witnessing sharing of ancestral polymorphisms between the species pairs $A n$. bwambae/An. merus and An. bwambae/An. quadriannulatus. 
Table 5: Likelihood ratio tests in NOS and Rel2 between models that allow codon sites to evolve under positive selection (M3, M2a, M8) and those that do not (MO, MIa, M7).

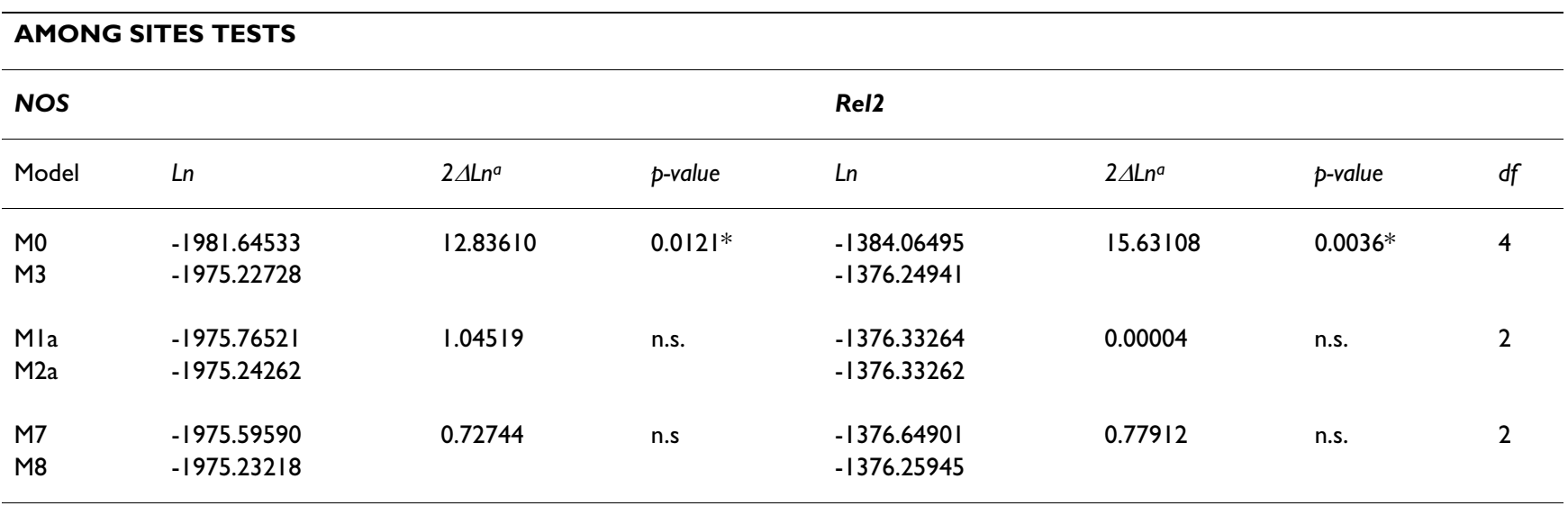

* Significant $p$-value at 0.05 significance level; $d f$ : degrees of freedom

a This quantity is compared to the critical values of a chi-square distribution with the respective degrees of freedom

We claim this since out of ten shared polymorphisms, four were shared between the previously mentioned pairs of species.

The expression pattern of NOS was found to vary depending on the Plasmodium species infecting An. gambiae [18], implying that the differential expression pattern is the effect of co-evolution between the host and its specific parasite. The present study does not add support to this interpretation since both the McDonald-Kreitman tests and the PAML analysis did not detect any signs of positive selection acting on the NOS gene. Even though the PAML analysis indicated that varying selective pressure is acting on the codons of the NOS fragment amplified (Table 5), all other likelihood models that allowed for positive selection to be acting on some codons or branches of the NOS fragment, produced negative results (Table 5, Table 6).

Table 6: Branch-site test 2 in NOS and Rel2 with An. arabiensis and An. gambiae designated as the foreground branches.

Branch-site test 2

NOS

\begin{tabular}{|c|c|c|c|c|}
\hline & Ln & $2 \Delta L n$ & p-value & $d f$ \\
\hline \multicolumn{5}{|c|}{ An. arabiensis: foreground branch } \\
\hline model $2, \omega=1$ & $-|780.2874| 5$ & 0.61390 & n.s. & 1 \\
\hline model $2, \omega$ free to vary & -1779.980464 & & & \\
\hline \multicolumn{5}{|c|}{ An. gambiae: foreground branch } \\
\hline model $2, \omega=1$ & -1779.79876 & 0.00000 & n.s. & 1 \\
\hline model $2, \omega$ free to vary & -1779.79876 & & & \\
\hline \multicolumn{5}{|l|}{ Rel2 } \\
\hline & $\operatorname{Ln}$ & $2 \Delta L n$ & p-value & $d f$ \\
\hline \multicolumn{5}{|c|}{ An. arabiensis: foreground branch } \\
\hline model $2, \omega=1$ & -1376.33322 & 0.00100 & n.s. & 1 \\
\hline model $2, \omega$ free to vary & -1376.33272 & & & \\
\hline \multicolumn{5}{|c|}{ An. gambiae: foreground branch } \\
\hline model $2, \omega=1$ & -1374.86019 & 0.41907 & n.s. & 1 \\
\hline model $2, \omega$ free to vary & -1374.65065 & & & \\
\hline
\end{tabular}

$d f$ degrees of freedom 
In the phylogenetic tree of Rel2 (Figure 1) there is a group of An. gambiae alleles that are very closely related to alleles of An. bwambae. Shared polymorphisms in the Rel2 locus are detected between An. arabiensis and An. gambiae but not between An. gambiae and An. bwambae. Consequently, the unexpected clustering of the An. gambiae alleles with An. bwambae, is more likely due to introgression between the two species, as noted above for other genes.

At the Rel2 locus the level of nucleotide differences among species (Table 4) were comparable to those observed in the NOS locus. However, at the Rel2 locus, there are several fixed non-synonymous differences between certain pair of species. For example, between An. arabiensis and An. melas there were eight non-synonymous fixed differences. Neither the McDonald-Kreitman nor the maximum likelihood tests for selection provide evidence of positive selection in this gene. Rel2, like gambicin and NOS was found to be under purifying selection. As was the case in NOS, the maximum likelihood analysis of PAML, pointed to a varying selective pressure among sites (M0 vs M3, pvalue $=0.0036$ : Table 5 ), but no signs of positive selection were detected in the site by site or the branch-site analysis (Table 5, Table 6).

Rel2 regulates the expression of the antibacterial genes CEC1, GAM1, DEF1, CEC3, and key malaria parasite antagonists, LRIM1, TEP1, and TEP4 in An. gambiae [17]. Thus, Rel2 is regulating the expression pattern of many and diverse genes of the Anopheles innate immune system, among which at least two (LRIM1 and TEP1) have very strong anti-malaria activity. However, the remaining genes regulated by Rel2 are, as far as known, antibacterial peptides. Therefore, even though Rel2 may be involved in the defense against malaria infection, its involvement in the expression pattern of many antimicrobial genes may greatly reduce the possibility of this gene specifically exhibiting an evolutionary response to Plasmodium. However the comparative approach applied here did detect purifying selection acting on the amplified Rel2 fragment from the six species of the An. gambiae, as would be expected in a locus coding for a product that serves as the regulator of the expression of the general antimicrobial defense of the organism. Because Rel2 is mostly involved in antibacterial defense, why are we not witnessing positive selection imposed by the bacterial pathogens? This type of positive selection has been reported for GNBP1, GNBP2 and Relish in termites and was related to the diverse microbes encountered in different habitats [27]. In the present study, there are significant ecological differences among the studied species. For example the species An. melas and An. merus favour brackish water for immature development, in contrast to the freshwater species $A n$. gambiae and An. arabiensis. Even though ecological differences among the studied species are evident, no positive selection could be detected. At this point we have to point that the Rel2 fragment targeted in our study resides in the N-terminal homology domain region of Rel2, whereas positive selection has been detected [27] in the spacer connecting this region to the C-terminal ankyrin repeat region (see 27).

The FBN9 locus is characterized by a very large number of polymorphic site differences (mainly synonymous) between the species (Table 4). In the phylogenetic tree of FBN9 (see Additional file 2) the alleles of An. gambiae are interspersed across the tree, whereas the alleles of the remaining species form strongly supported monophyletic groups ambiguously connected to each other. Once more the observed pattern could be the result of introgression between species, or the sharing of ancestral polymorphisms. Because the shared polymorphisms are present in almost all species pairwise comparisons in the case of FBN9, we argue that the effect of shared ancestral polymorphisms has played a key role in this pattern. In some cases the shared polymorphisms are as many as eight ( $A n$. arabiensis-An. bwambae) or 19 (An. arabiensis-An. gambiae).

Characterization of the FBN gene family has suggested that the FBNs have structural features (pattern recognition receptors) that allow them to recognize parasites and play an essential role in the mosquito's innate immunity, in addition to the physiological processes associated with blood feeding [28]. FBN9 a member of the FBN family, was recently found to be up-regulated when An. gambiae was invaded by $P$. falciparum ookinetes but not responding to $P$. berghei ookinetes invasion [11]. The structural features of FBN9, as well as the fact that in RNAi gene silencing assays, a FBN9 knockdown increases An. gambiae mosquitoes susceptibility to both $P$. falciparum and $P$. berghei infections [11], raise the possibility that this gene could be evolving in response to Plasmodium. Furthermore, in a recent study [29] it was shown that positive selection drives the evolution of pattern recognition receptors in Drosophila. However, even in the case of FBN9 no signs of positive selection could be detected and there were not even any fixed non-synonymous differences between the species (Table 4). FBN9 is also involved in the immune response against bacteria [11] and this could be the major reason why this gene as well has not undergone major adaptations to malaria.

Presently there are only three studies assessing genetic variation in innate immunity genes specifically implicated as being important in controlling Plasmodium infection in Anopheles [20,30,31]. In a study of the defensin gene [31] An. gambiae, An. arabiensis and An. quadriannulatus were involved and the authors concluded that strong purifying selection is acting on the mature peptide and probably the whole coding region. Furthermore, the authors argued 
that since An. quadriannulatus is not exposed to human pathogens, identical mature peptide and similar pattern of polymorphism across the three species implies that human pathogens played no role as selective agents on this peptide. Similarly, it was concluded [30] that no evidence for strong selection could be detected on a suite of mosquito immune system genes, CTL4, CTLMA2, LRIM1, and APL2 (or LRRD7), which have been shown to affect Plasmodium development in functional studies. The authors used five different species of the An. gambiae complex, namely An. gambiae, An. arabiensis, An. bwambae and An. merus. However, they only focused on An. gambiae and used all remaining species as outgroups in their analyses. One of the loci studied in [30], LRIM1, has been the subject of a separate study [20] in which besides the conservative McDonald-Kreitman tests for positive selection, a maximum likelihood approach with PAML software was also applied. In the latter study, six species of the An. gambiae complex were involved and it was concluded that LRIM1 underwent adaptive evolution in the An. arabiensis lineage.

LRIM1 was recently established as a major anti-Plasmodium factor [15] and it has been speculated that it could be possible that only some LRIM1 alleles suppress infection with $P$. falciparum, and these may even be specific for certain P.falciparum strains [20]. LRIM1 is the only gene of the Anopheles immune system that directly interacts with Plasmodium and has not been implicated to be involved in the defense against other pathogens. LRIM1 is also the only Anopheles immune gene that has been studied that shows strong signs of positive selection acting in one of the major malaria vectors, An. arabiensis [20].

\section{Conclusion}

It is not surprising that studies of the genes considered here as well as those by [31] and [30] could not detect selection related specifically to Plasmodium. However, the positive results of [20] lend credence to the general comparative approach in identifying the minority of the hundreds of genes implicated in the insect innate immunity response that may have responded specifically to Plasmodium. Considering the results presented here in concert with previous studies, genes that interact directly with the Plasmodium parasite, and play little or no role in defense against other microbes, are probably the most likely candidates for a specific adaptive response against $P$. falciparum. However, even though several lines of evidence exist and support that that the Anopheles species have exhibited an adaptive response to $P$. falciparum infection [20], we cannot completely exclude the possibility that the mosquitoes are utilizing solely their anti-bacterial defense system to fight against Plasmodium. Furthermore, since it is hard to establish direct evidence linking the adaptation of any candidate gene to $P$. falciparum infec- tion, a comparative framework allowing at least an indirect link should be provided. Such a framework could be achieved, if a similar approach like the one involved here, was applied to all other anopheline complexes that transmit P. falciparum malaria. Such complexes do exist (i.e. Anopheles funestus, Anopheles nili, Anopheles moucheti) in continental sub-Saharan Africa. Considering the systematic status of these anopheline complexes [32,33], it can be safely argued that the acquisition of $P$. falciparum by each of these complex of species was an independent evolutionary event. In the case that the same candidate gene and/or the same fragment of the respective gene, was identified as being evolving under positive selection in more than one of these complexes, then an indirect yet very strong proof of evolution imposed by $P$. falciparum infection, would be recognized.

\section{Methods \\ Mosquitoes sampling}

Six species of the An. gambiae species complex were included in this study. The adult An. gambiae specimens used were collected from two regions of Cameroon (Mbebé and Nyabéssan). Adult An. arabiensis females were collected from Kousseri in Cameroon, whereas adult An. melas were collected from Ipono (Cameroon). Larvae of An. bwambae originating from Bwamba county in Uganda, were kindly provided by Ralph Harbach. DNA extracts of An. merus were kindly provided by David O' Brochta (collected from Furvela in Mozambique). Finally, An. quadriannulatus A specimens from Kruger National Park in South Africa, were kindly provided by Anton Cornel.

\section{DNA methods}

DNA was extracted using the DNeasy tissue kit (Qiagen) using either the entire mosquito or 2-3 of its legs. Species and molecular form identification was performed following recent diagnostic protocols [34,35]. All An. gambiae specimens belonged to the $\mathrm{S}$ molecular form.

\section{Loci analysed}

We amplified four An. gambiae loci that have been experimentally associated with malaria infection. Multiple primers were designed for each one of the targeted loci using the software FastPCR [36] and based on the An. gambiae genome [3]. The amplified genes were: a) Gambicin (Ensembl Gene Id: AGAP008645, Chromosome 3R) that is composed of three exons and has a total length of 648 bp. Of these only 246 bp are coding sequence. Gambicin is an antimicrobial peptide that is ultimately secreted as a 61-aa mature peptide. It is induced during both early and late stages of malaria infection. In vitro experiments showed [19] that the mature peptide can kill both gram-positive and gram-negative bacteria, has a morphogenic effect on a filamentous fungus, and is mar- 
ginally lethal to Plasmodium berghei ookinetes. The primer pairs designed in this study targeted the whole coding region of the gene and the introns in between. b) Nitric Oxide Synthase (NOS) [Ensembl Gene Id: AGAP008255, Chromosome $3 \mathrm{R}$ ] which is a complex gene, and is comprised of 18 exons separated by introns of varying length and spread over about $33 \mathrm{~kb}$ (exons: $3342 \mathrm{bp}$ ). It was recently suggested [18] that $P$. falciparum ingestion triggers a midgut-associated, as well as a systemic, response in the mosquito, involving three genes, one of which is NOS. We targeted a fragment of approximately 1000 bp (part of exon 14, exons 15 and 16, part of exon 17 and all the introns between those exons). c) Rel2 (Ensembl Gene Id: AGAP006747, Chromosome 2L) that is comprised of 10 exons separated by introns of varying size. The total length of the gene is approximately $11.66 \mathrm{~kb}$ out of which only $3779 \mathrm{bp}$ belong to exons. It has been found that this gene is involved [17] in the regulation of the intensity of mosquito infection with the malaria parasite, $P$. berghei. The primers used amplified a fragment of approximately 800 bp, including parts of exons 3 and 4 and the intron in between. d) FBN9 (Ensembl Gene Id: AGAP011197, Chromosome $3 \mathrm{~L}$ ) that is a single exon gene ( $846 \mathrm{bp}$ ) and produces a 282-aa mature peptide. FBN9 has been found to be up-regulated in An. gambiae after $P$. falciparum infection, but not with $P$. berghei infection [11]. The primers designed in this study aimed for a fragment of $810 \mathrm{bp} \mathrm{cov}-$ ering almost the complete gene.

In most of the cases a nested PCR protocol was applied to successfully amplify the targeted loci. In the nested PCR protocol, the product of a PCR using a specific set of primers was used as a template for a subsequent PCR using primers internal to the ones used in the preceding PCR. The sequences of the primers used in the amplification of each locus are reported in Table 1. PCR products were examined on a $2 \%$ agarose gel, purified using the Qiaquick Purification Kit (Qiagen) and submitted for direct sequencing. The PCR products were sequenced in both directions using BigDye ${ }^{\mathrm{TM}}$ Terminator Cycle Sequencing Kit (v3.1, Applied BioSystems) reagents and an 3730 ABI capillary sequencer. All individuals that were found to be heterozygous for two or more positions were subjected to PCR amplification again and the amplicons were cloned using the TOPO-TA cloning kit for sequencing (Invitrogen). From each individual, a minimum of three transformed colonies were selected, and the size of the DNA insert was screened by PCR using the T3/T7 primer pair of the TOPO-TA vector. In most of the cases the correct size insert was obtained, and was subsequently sequenced in both directions. Because of multiple insertion/deletion (indels) in the introns of the gambicin locus, direct sequencing usually produced sequences of low quality. In order to circumvent this issue, most of the gambicin sequences produced were obtained via cloning of the
PCR products. In this case, a minimum of five individually transformed colonies from each individual were screened, and at least three were sequenced. In all PCRs to ensure the minimum number of miss-incorporations Platinum High Fidelity Taq (Invitrogen) was used.

All produced sequence chromatograms were inspected by eye to confirm the validity of all differences either between alleles of the same individual, or within and between species. Sequences were viewed, edited and assembled using CodonCode Aligner (v. 1.6.3 CodonCode Corporation, Dedham, MA, USA). All produced sequences were compared to the published An. gambiae genome [3] to verify their homology to the respective loci. Sequences produced for this study have been submitted to GenBank under the accession numbers EU304549 to EU304787.

\section{Species polymorphism and divergence}

All sequences were aligned using CodonCode Aligner (v. 1.6.3 CodonCode Corporation, Dedham, MA, USA). Basic analyses of polymorphism and divergence were performed using the computer program DNAsp v.4.10.3 [37]. Parameters estimated included the pairwise diversity (Pi) at synonymous and non-synonymous sites and the average number of nucleotide substitutions per site between species (Dxy).

\section{Tests for selection}

In order to assess whether selection is acting on any of the immunity genes amplified from the six species of the An. gambiae complex, two different approaches were implemented. The first approach involved the McDonald-Kreitman test [38] that is intended to identify selection through an excess of amino acid substitution between species. This test compares the $\mathrm{dN} / \mathrm{dS}$ ratio between species to within species and is based on the idea that substitutions under positive selection will go to fixation rapidly, and are therefore rarely observed as polymorphisms. However, they are present as fixed differences between species and an excess of replacement fixed differences is therefore an indication of positive selection. This test allows the detection of selection on a whole protein is bound to be quite conservative in detecting selection [39] and lacks the power of a site by site analysis. This analysis was performed using DNAsp v.4.10.3 [37].

A second and more powerful method to detect selection was also applied. This method detects elevated $\mathrm{dN} / \mathrm{dS}$ ratios ( $\omega$ ratios) using maximum likelihood approaches (see Yang 2007) and is less conservative than the McDonald-Kreitman test. We reasoned that it may be hard to detect positive selection on the whole of each amplified fragment, because the majority of their codons are likely to be functionally constrained and therefore under purify- 
ing selection. However, such purifying selection may be masking positive selection of a small number of codons within the amplified fragments. We therefore used a codon by codon maximum likelihood test, to ask if we could detect any codons that have been under repeated, strong positive selection. This method allows a site by site analysis, thus the identification of particular codons that have been evolving under selective constrains [40] is feasible. This analysis was performed using the software package PAML v. 4. [41]. At this point we have to stress that since we are dealing with a set of species very closely related, one has to be cautious in the interpretation of the results and the attribution of unusual patterns of diversity to selection. It is certain that shared ancestral polymorphisms and recent introgression between the complex members, will confound the actual processes. The loci subjected to the PAML analysis were those that exhibited at least one fixed non-synonymous change (i.e. NOS and Rel2), in some of the pairwise species comparisons (Tables 3, 4). Each locus was separately analyzed. Using sequence data from the coding and non-coding region of each amplified locus, a gene tree describing the phylogenetic relationships of all the taxa studied, was generated. The trees were constructed with the phylogenetic software program MrBayes 3.1 [42], using partitioned data. The data sets were partitioned so that a different substitution model could be applied to the introns, the first, second, and third codon positions of each gene. The substitution models implemented for each partition in the Bayesian analysis, were those suggested by Modeltest 3.7 [43] according to the Akaike Information Criterion [44]. The generated Bayesian trees served as the basis for the implementation of the maximum likelihood methods of the PAML package of programs [41] aiming at detecting adaptive molecular evolution under specific models of codon substitution. When sequences evolve under neutrality, the relative number of synonymous and non-synonymous substitutions is expected to be 1 . In the case of positive selection, amino acid changes are favored and $\omega>1$, whereas under purifying selection amino acid changes are prevented and $\omega<1$.

In each one of the loci subjected to the PAML analysis, we estimated the likelihood values of the respective phylogenetic tree as being the result of lineages evolving under the assumptions of the site models M0, M3, M1a, M2a, M7 and M8 implemented in PAML. These models allow $\omega$ values to vary among different codons. Following the suggestions of Yang (2007) the site model pairs that appear to be particularly useful for real data analysis, are the M1a versus M2a and M7 versus M8. However, we also compared model M0 versus M3 in order to see if the selective pressure is uniform among sites. The strength of positive selection was calculated by comparing twice the log likelihood difference in a chi-square test with four (M0 versus $\mathrm{M} 3$ ) or two (M1a versus M2a, and M8 versus M7) degrees of freedom.

Finally, aiming at investigating whether the branches leading to the two major human malaria vectors, An. gambiae and An. arabiensis, are evolving under positive selection, we applied the branch-site models for each one of this species. Thus, the An. gambiae lineage in one case and the An. arabiensis lineage in the other case, were considered as the foreground branches (branch evolving with an $\omega$ value different than one), and $\omega$ values were allowed to vary among lineages and among sites [40]. Model 2, with $\omega$ free to vary (model $=2$, several $\omega$ values for branches: settings of PAML), was compared to the same model, but with $\omega$ fixed to one (branch-site test 2) in order to examine if indeed the $\omega$ value of the foreground lineage is significantly different from one. The strength of positive selection was calculated by comparing twice the log likelihood difference in a chi-square test with 1 degree of freedom.

\section{Authors' contributions}

AP produced the genetic data, carried out the analyses, and wrote the manuscript. JRP, and AC helped drafting the manuscript. JRP, AC and AP conceived and designed the experiments. JRP and AC coordinated the project. MAS, JCM, FS, PHA, CAN contributed reagents/materials and analysis tools. All authors read and approved the final manuscript.

\section{Additional material}

\section{Additional file 1}

Gambicin Bayesian Inference Tree. 50\% majority-rule consensus Bayesian (unrooted) tree of gambicin. Numbers on branches are the posterior probabilities of clades, only values above 0.5 are presented. Species names have been abbreviated as follows: ARA: An. arabiensis, BWA: An. bwambae, GAM: An. gambiae, MEL: An. melas, MER: An. merus, and QUA: An. quadriannulatus. The number following the species abbreviation refers to the individual specimen code, whereas the letters $A$ and $B$ differentiate between the two alleles of a single individual specimen. Details of the Bayesian analysis can be provided upon request.

Click here for file

[http://www.biomedcentral.com/content/supplementary/14712148-8-79-S1.doc] 


\section{Additional file 2}

FBN9 Bayesian Inference Tree. 50\% majority-rule consensus Bayesian (unrooted) tree of FBN9. Numbers on branches are the posterior probabilities of clades, only values above 0.5 are presented. Species names have been abbreviated as follows: ARA: An. arabiensis, BWA: An. bwambae, GAM: An. gambiae, MEL: An. melas, MER: An. merus, and QUA: An. quadriannulatus. The number following the species abbreviation refers to the individual specimen code, whereas the letters $A$ and $B$ differentiate between the two alleles of a single individual specimen. Details of the Bayesian analysis can be provided upon request.

Click here for file

[http://www.biomedcentral.com/content/supplementary/14712148-8-79-S2.doc]

\section{Acknowledgements}

We are very grateful to Anton Cornel, Ralph Harbach and David O'Brochta for providing An. quadriannulatus A, An. bwambae and An. merus specimens, respectively. We also wish to express our gratitude to George Dimopoulos and George Christophides for constructive comments and suggestions on issues relating to the choice of immune genes to be studied. This research was supported by the National Institutes of Health grant ROI AI 046018 to J.R.P and A.C. Additionally, A.P. was supported by a Marie Curie Outgoing International Fellowship (Contract No. MOIF-CT-2006-02 I357).

\section{References}

I. Takken W, Boëte C: An introduction to ecological challenges concerning the use of genetically-modified mosquitoes for disease control. In Ecological aspects for application of genetically modified mosquitoes Volume 2. Dordrecht, Netherlands: Kluwer Academic Publishers; 2003:9-12.

2. Aylward B, Hennessey KA, Zagaria N, Olive JM, Cochi S: When is a disease eradicable? 100 years of lessons learned. American Journal of Public Health 2000, 90(10): $1515-1520$.

3. Holt RA, Subramanian GM, Halpern A, Sutton GG, Charlab R, Nusskern DR, Wincker P, Clark AG, Ribeiro JMC, Wides R, et al:: The Genome Sequence of the Malaria Mosquito Anopheles gambiae. Science 2002, 298(559 I): I29-149.

4. Nene V, Wortman JR, Lawson D, Haas B, Kodira C, Tu ZJ, Loftus B, Xi ZY, Megy K, Grabherr M, et al: Genome sequence of Aedes aegypti, a major arbovirus vector. Science 2007, 316(5832): $1718-1723$.

5. James AA: Blocking malaria parasite invasion of mosquito salivary glands. Journal of Experimental Biology 2003, 206(21):3817-3821.

6. Jacobs-Lorena $\mathrm{M}$ : Interrupting malaria transmission by genetic manipulation of anopheline mosquitoes. Journal of Vector Borne Diseases 2003, 40(3-4):73-77.

7. Dinglasan RR, Kalume DE, Kanzok SM, Ghosh AK, Muratova O, Pandey A, Jacobs-Lorena M: Disruption of Plasmodium falciparum development by antibodies against a conserved mosquito midgut antigen. Proceedings of the National Academy of Sciences of the United States of America 2007, 104(33): 1346 I-13466.

8. Nirmala X, James AA: Engineering Plasmodium-refractory phenotypes in mosquitoes. Trends in Parasitology 2003, I 9(9):384-387.

9. Zieler $\mathrm{H}$, Keister DB, Dvorak JA, Ribeiro JMC: A snake venom phospholipase $A(2)$ blocks malaria parasite development in the mosquito midgut by inhibiting ookinete association with the midgut surface. Journal of Experimental Biology 200I, 204(23):4157-4167.

10. Abraham EG, Pinto SB, Ghosh A, Vanlandingham DL, Budd A, Higgs $\mathrm{S}$, Kafatos FC, Jacobs-Lorena M, Michel K: An immune-responsive serpin, SRPN6, mediates mosquito defense against malaria parasites. Proceedings of the National Academy of Sciences of the United States of America 2005, 102(45): 16327-16332.
II. Dong YM, Aguilar R, Xi ZY, Warr E, Mongin E, Dimopoulos G: Anopheles gambiae immune responses to human and rodent Plasmodium parasite species. Plos Pathogens 2006, 2(6):5 I3-525.

12. Dong YM, Taylor HE, Dimopoulos G: AgDscam, a hypervariable immunoglobulin domain-containing receptor of the Anopheles gambiae innate immune system. Plos Biology 2006, 4(7): I |37-I| 46.

13. Michel K, Budd A, Pinto S, Gibson TJ, Kafatos FC: Anopheles gambiae SRPN2 facilitates midgut invasion by the malaria parasite Plasmodium berghei. Embo Reports 2005, 6(9):89|-897.

14. Michel K, Kafatos FC: Mosquito immunity against Plasmodium. Insect Biochemistry and Molecular Biology 2005, 35(7):677-689.

15. Osta MA, Christophides GK, Kafatos FC: Effects of mosquito genes on Plasmodium development. Science 2004, 303(5666):2030-2032.

16. Vlachou D, Kafatos FC: The complex interplay between mosquito positive and negative regulators of Plasmodium development. Current Opinion in Microbiology 2005, 8(4):4I5-42I.

17. Meister S, Kanzok SM, Zheng XL, Luna C, Li TR, Hoa NT, Clayton JR, White KP, Kafatos FC, Christophides GK, et al.: Immune signaling pathways regulating bacterial and malaria parasite infection of the mosquito Anopheles gambiae. Proceedings of the National Academy of Sciences of the United States of America 2005, I 02(32): I | 420-I I 425

18. Tahar R, Boudin C, Thiery I, Bourgouin C: Immune response of Anopheles gambiae to the early sporogonic stages of the human malaria parasite Plasmodium falciparum. Embo Journal 2002, 2 I (24):6673-6680.

19. Vizioli J, Bulet P, Hoffmann JA, Kafatos FC, Muller HM, Dimopoulos G: Gambicin: A novel immune responsive antimicrobial peptide from the malaria vector Anopheles gambiae. Proceedings of the National Academy of Sciences of the United States of America 200 I, 98(22): $12630-12635$

20. Slotman M, Parmakelis A, Marshall J, Awono-Ambene P, AntonioNkondjo C, Simard F, Adalgisa C, Powell J: Patterns of Selection in Anti-Malarial Immune Genes in Malaria Vectors: Evidence for Adaptive Evolution in LRIMI in Anopheles arabiensis. PLoS One 2007, 2(8):e793

21. Besansky NJ, Krzywinski J, Lehmann T, Simard F, Kern M, Mukabayire O, Fontenille D, Toure Y, Sagnon NF: Semipermeable species boundaries between Anopheles gambiae and Anopheles arabiensis: Evidence from multilocus DNA sequence variation. Proceedings of the National Academy of Sciences of the United States of America 2003, 100(19):10818-10823.

22. Slotman MA, Della Torre A, Calzetta M, Powell JR: Differential introgression of chromosomal regions between Anopheles gambiae and An. arabiensis. American Journal of Tropical Medicine and Hygiene 2005, 73(2):326-335.

23. Gentile G, della Torre A, Maegga B, Powell JR, Caccone A: Genetic differentiation in the African malaria vector, Anopheles gambiae ss, and the problem of taxonomic status. Genetics 2002, 16I(4): I56I-1578.

24. Kimura M: A simple method for estimating evolutionary rates of base substitutions through comparative studies of nucleotide-sequences. Journal of Molecular Evolution 1980 , 16(2): III-120.

25. Kumar S, Gupta L, Han YS, Barillas-Mury C: Inducible Peroxidases mediate nitration of Anopheles midgut cells undergoing apoptosis in response to Plasmodium invasion. Journal of Biological Chemistry 2004, 279(5 I):53475-53482.

26. Thelwell NJ, Huisman RA, Harbach RE, Butlin RK: Evidence for mitochondrial introgression between Anopheles bwambae and Anopheles gambiae. Insect Molecular Biology 2000, 9(2):203-210.

27. Bulmer MS, Crozier $\mathrm{RH}$ : Variation in positive selection in termite GNBPs and relish. Molecular Biology and Evolution 2006, 23(2):317-326.

28. Wang $X$, Zhao Q, Christensen B: Identification and characterization of the fibrinogen-like domain of fibrinogen-related proteins in the mosquito, Anopheles gambiae, and the fruitfly, Drosophila melanogaster, genomes. BMC Genomics 2005, 6(I): 114

29. Sackton TB, Lazzaro BP, Schlenke TA, Evans JD, Hultmark D, Clark $A G$ : Dynamic evolution of the innate immune system in Drosophila. Nature Genetics 2007, 39( I 2): | 46 |-| 468. 
30. Obbard DJ, Linton YM, Jiggins FM, Yan G, Little TJ: Population genetics of Plasmodium resistance genes in Anopheles gambiae: no evidence for strong selection. Molecular Ecology 2007, I 6(16):3497-35I0.

31. Simard F, Licht M, Besansky NJ, Lehmann T: Polymorphism at the defensin gene in the Anopheles gambiae complex: Testing different selection hypotheses. Infection Genetics and Evolution 2007, 7(2):285-292.

32. Harbach RE: Review of the internal classification of the genus Anopheles (Diptera, Culicidae) - The foundation for comparative systematics and phylogenetic research. Bulletin of Entomological Research 1994, 84(3):33 I-342.

33. Harbach RE: The classification of genus Anopheles (Diptera : Culicidae): a working hypothesis of phylogenetic relationships. Bulletin of Entomological Research 2004, 94(6):537-553.

34. Besansky NJ, Collins FH, Townson H: A species-specific PCR for the identification of the malaria vector Anopheles bwambae. Annals of Tropical Medicine and Parasitology 2006, I00(3):277-280.

35. Fanello C, Santolamazza F, della Torre A: Simultaneous identification of species and molecular forms of the Anopheles gambiae complex by PCR-RFLP. Medical and Veterinary Entomology 2002, 16(4):46I-464.

36. Kalendar R: FastPCR: a PCR primer and probe design and repeat sequence searching software with additional tools for the manipulation and analysis of DNA and protein. Helsinki, Finland; 2007.

37. Rozas J, Sanchez-DelBarrio JC, Messeguer X, Rozas R: DnaSP, DNA polymorphism analyses by the coalescent and other methods. Bioinformatics 2003, 19:2496-2497.

38. McDonald JH, Kreitman M: Adaptive protein evolution at the adh locus in Drosophila. Nature I991, 35 I(6328):652-654.

39. Nielsen R: Statistical tests of selective neutrality in the age of genomics. Heredity 200I, 86:64I-647.

40. Yang ZH, Bielawski JP: Statistical methods for detecting molecular adaptation. Trends in Ecology \& Evolution 2000, I 5(12):496-503.

4I. Yang ZH: PAML 4: Phylogenetic analysis by maximum likelihood. Molecular Biology and Evolution 2007, 24(8): I586-159I.

42. Ronquist F, Huelsenbeck JP: MrBayes 3: Bayesian phylogenetic inference under mixed models. Bioinformatics 2003, I (1 (2): I572-1574.

43. Posada $D$, Crandall KA: MODELTEST: testing the model of DNA substitution. Bioinformatics 1998, 14(9):817-818.

44. Akaike H: New look at statistical-model identification. leee Transactions on Automatic Control 1974, Ac 1 9(6):716-723.

Publish with Bio Med Central and every scientist can read your work free of charge

"BioMed Central will be the most significant development for disseminating the results of biomedical research in our lifetime. "

Sir Paul Nurse, Cancer Research UK

Your research papers will be:

- available free of charge to the entire biomedical community

- peer reviewed and published immediately upon acceptance

- cited in PubMed and archived on PubMed Central

- yours - you keep the copyright
BioMedcentral 